\title{
LA TRANSFORMACIÓN Y DISRUPCIÓN DE LAS TECNOLOGÍAS DE LA INFORMACIÓN Y LAS POLÍTICAS ECONÓMICAS DE COLOMBIA COMO PARTE DE LA GLOBALIZACIÓN EMPRESARIAL
}

\section{THE TRANSFORMATION AND DISRUPTION OF INFORMATION TECHNOLOGIES AND ECONOMIC POLICIES OF COLOMBIA AS PART OF BUSINESS GLOBALIZATION}

\author{
Esp. Deisy Esthér Castro Márquez.* \\ Esp. Jorge Alberto Camargo Barbosa.** \\ Dr. Hugo Fernando Castro Silva \\ * Universidad Francisco de Paula Santander, Facultad de Ingeniería, Programa de \\ Maestría en Gobierno de Tecnología de Información, Grupo de Investigación \\ GITYD. \\ Vía Acolsure Sede el Algodonal, Ocaña, Norte de Santander, Colombia. \\ Teléfono, (57) (7)5690088 \\ E-mail:info@ufpso.edu.co. \\ **Facultad de Sogamoso, Universidad Pedagógica y Tecnológica de Colombia, \\ Sogamoso, Colombia. hugofernando.castro@uptc.edu.co
}

\begin{abstract}
Resumen: Este documento da un opinión sobre la transformación y disrupción de las tecnologías de la información y las políticas económicas de Colombia como parte de la globalización empresarial que actualmente se vive en el país en razón a ser más competitivos y sostenibles frente a la misma, a partir de un enfoque holístico de las tecnologías en las organizaciones en nuestra región. De esta manera, se describen políticas económicas y sociales asociadas a la tecnología, el apoyo del estado colombiano y los aspectos relevantes que dan respuesta a sobre los cambios, impactos, beneficios y perjuicios de la dinámica de la globalización y las TI en Colombia.
\end{abstract}

Palabras clave: Transformación, Disrupción, Globalización, Cambios, Impactos, Beneficios, Debilidades, Tecnologías de la Información.

Abstract: Our This document gives an opinion on the transformation and disruption of information technologies and the economic policies of Colombia as part of the business globalization that is currently being lived in the country, in order to be more competitive and sustainable compared to it, starting of a holistic approach to technologies in organizations in our region. In this way, economic and social policies associated with technology, the support of the Colombian state and the relevant aspects that respond to the changes, impacts, benefits and damages of the dynamics of globalization and IT in Colombia are described.

Keywords: Transformation, Disruption, Globalization, Changes, Impacts, Benefits, Weaknesses, Information Technologies. 


\section{INTRODUCCIÓN}

La globalización hoy en día exige que las organizaciones y en especial los países adopten nuevas maneras de enfrentar las necesidades de la sociedad. Es así, que en el contexto empresarial se ve sometida a la adopción e implementación de herramientas tecnológicas, el cual contribuyen al desarrollo y evolución de las organizaciones y de los países. En este caso, lograr incursionar y mantenerse al nivel del mercado mundial resulta un reto e inversión de grandes recursos económicos, sociales, políticos y culturales. La globalización cada día exige más especialidad, calidad, seguridad, tecnología y buenas prácticas sobre las empresas y los países que piensan en surgir, desarrollarse y continuar evolucionando al ritmo de las exigencias del mercado. Esto conlleva a pensar el camino que la empresa colombiana, y en especial el país, hace en razón de poder ser competitivos y sostenibles frente a los requerimientos de una globalización que cada día se posiciona como un eslabón en los negocios que requiere ser fortalecidos. Con esta motivación, en este estudio se pretende dar una opinión a que si la empresa colombiana y nuestro país está preparado para la disrupción tecnológica que trae la globalización como base fundamental que impulsa a ser competitivos y sostenibles en el mercado mundial?

\section{GLOBALIZACIÓN}

"La globalización es el reconocimiento por parte de las organizaciones que deben tener un enfoque global y no un enfoque local. A nivel mundial, la globalización se refiere a la creciente interdependencia entre los países. Es un proceso de crecimiento internacional o mundial del capital financiero, industrial, comercial, recursos, humano, político y de cualquier tipo de actividad intercambiable entre países" (Barroso, Paola. Globalización. 2009).

La globalización tiene diferentes impactos sobre los mercados internacionales, este impacto está ligado a las características de los diferentes sectores, el desarrollo económico, social, político, cultura, productivo y tecnológico, de las regiones, las necesidades y expectativas de la población (Henao, Laura. 2013).
La Globalización en los mercados mundiales hoy en día depende en gran parte del uso de la tecnología. Dichas herramientas tecnológicas inicialmente pudieron ser concebidas como instrumentos de apoyo para las organizaciones con fines de optimizar sus procesos en razón de tiempo, procesamiento, almacenamiento, entre otras características. Sin embargo, con el tiempo la necesidad de comunicarse y transmitir información a distancias "impensables", en un entonces, que ya hacen parte del pasado, despertó el interés en el uso de las telecomunicaciones como parte importante para logra enlazar, compartir y transmitir información a nivel regional, nacional y mundial, considerando la posibilidad de abrir los mercados a gran parte del planeta. Así mismo, esta revolución tecnológica ha hecho que cada día aumenten los mercados, y a su vez, las amenazas informáticas que hoy en día son una realidad y un riesgo emergente que ha tomado importancia en su tratamiento, con el fin de proteger los negocios.

\section{TRANSFORMACIÓN Y DISRUPCIÓN}

Es evidente que los países desarrollados, debido a los grandes ingresos y disponibilidad económica tienen la posibilidad de adquirir e implementar más recursos tecnológicos, razón por la cual cada día se hacen más robustos en el campo empresarial y en el mercado internacional, siendo competitivos y sostenibles. Contradictorio a esto, los países que no son desarrollados invierten mucho menos en recursos tecnológicos debido a sus límites que poseen frente a ingresos o utilidades propias. Esto hace que la desigualdad, sea cada vez más notoria entre los países más desarrollados con aquellos que luchan por sobresalir de alguna manera en el campo empresarial. Sin embargo, es necesario que los países desarrollados mantengan una conciencia colectiva en el desarrollo mundial especialmente a nivel tecnológico, ya que de esto depende de alguna manera que su oferta de servicios a nivel internacional se vea aumentada o por lo menos estable en el mercado. Por esa razón, pensar en tener el poder tecnológico a nivel empresarial interno no siempre es beneficioso, ya que disponer de tecnología no garantiza la oportunidad de mantener una igualdad entre naciones que las poseen y de aquellas que no disponen de las mismas; es así, como puede ser visto la relación entre un país como Estados Unidos y Haití, donde 
prevalece para este último la inversión de recursos en el bienestar de la sociedad a nivel alimentario, dejando de lado la posibilidad de invertir recursos a nivel tecnológico como una oportunidad de disminución de la pobreza, aumento de empleo a través de tecnologías emergentes, y en efecto, el desarrollo como país. Por esta razón, es importante que las naciones desarrolladas piensen y actúen en apoyar a aquellas que requieren además de un apoyo social, también de asignación de recursos para que logren acercarse a una incursión tecnológica que les permita concebirse a este entorno que trae la globalización. Así mismo, por parte de los países en vía de desarrollo deben continuar procurando en la inversión de una cantidad de recursos significativos en la implementación de tecnologías a nivel empresarial como parte de su evolución y participación en un mercado internacional que le permita ser visible y adaptable a la economía mundial. Esto acompañado de un plan nacional y leyes que direccionen igualmente a las empresas a la implementación de tecnologías en su desarrollo corporativo. De igual manera, incentivos que motiven a las empresas a la adopción de tecnologías que permitan realizar estos cambios en el campo empresarial nacional, y en efecto, a nivel internacional.

Inevitablemente, hablar hoy de globalización implica tener presente una concepción tecnológica para las organizaciones y para los países. Esto conlleva, a pensar en una gran transformación y disrupción de las tecnologías que exige el mercado a nivel mundial debido a la disrupción que se está presentando. La transformación tecnológica puede incorporar directamente algunos términos relacionados con adquisición, innovación, cambio, adaptación, entre otros, referentes a la tecnología. Sin embargo, es importante diferenciar que disponer de tecnologías debe ser complementada con la transformación que las mismas puedan impactar en las organizaciones y en los países, a partir del conocimiento, adaptación y cultura sobre el uso de las mismas con un propósito establecido que se encuentre alineado con lo que quiere la organización y en lo que requiere el país incursionar a nivel mundial. Según Clayton Christensen, la innovación tecnológica disruptiva, se explica mejor si se la compara con las tecnologías de sostenimiento, que son las que mejoran las prestaciones de lo ya existente. Los avances tecnológicos de sostenimiento pueden ser radicales o incrementales pero se aplican en modelos que ya existen, mientras que las tecnologías disruptivas emergen. (Christensen,
Clayton. The innovator's dilemma". Harvard Business Review Press. 1997). Las Disrupciones tecnológicas dentro un proceso de cambio continúo hablan que la tecnología disruptiva genera nuevos productos y servicios, pero está vinculada al surgimiento también de nuevos tipos de consumidores, por eso, al menos al comienzo, no tienen por qué suponer una mejora de las prestaciones de lo que ya existe. Tiene que hacerlo de forma diferente. La disrupción se produce en el mercado, no en la tecnología. Nace una nueva propuesta de valor, nuevos clientes y nueva red de valor cuyos efectos se sienten en el mercado (Bruno Estrada López, 2016). Así mismo, respecto a la disrupción tecnológica a nivel comercial nos permitimos mencionar una definición sobre el término manifestada por el Gerente General de Oracle el cual dice que la disrupción tecnológica es transformar drásticamente la forma de hacer negocios (Arias, Jorge).

Hoy en día prácticamente todos los campos de acción en los que actúa la sociedad incentiva la aplicación de las tecnologías como una transformación de los mismos de una manera disruptiva. Algunos casos de transformación y disrupción tecnológica aplicada a diversos campos, puede evidenciarse en la tecnificación de la agricultura como lo trata Clayton J Torres, John F Archila, Mario L Tronco, Marcelo Becker, Arthur J Viera Porto, Alexander J Tiberti (2013), en su estudio cinemático de una plataforma robótica para agricultura. En el campo de la educación a nivel tecnológico Algemiro A. Ávila G, Elkin G. Flórez Serrano, Oscar Gualdrón G (2012) manifiesta el diseño e implementación de un curso interactivo multimedia para el aprendizaje de los procesos. Así mismo, Luis A Mesa Mesa, Nelson Barrera Lombana (2013) incluye la robótica educativa como instrumento didáctico alternativo en educación básica. Jaime Guzmán-Luna, IngridDurley Torres, Juan Felipe Alvarez (2014) define propone un generador de aplicaciones educativas basadas en televisión digital usando arquitectura de cómputo en la nube. Luis Fernando Gélvez R (2012) aplica redes neuronales morfológicas al reconocimiento de vocablos simples.

Considerando lo manifestado, surge la inquietud en cómo los países tratan de incursionar en la globalización que conlleva a una transformación y disrupción de las tecnologías en sus organizaciones y a nivel de estado. Para los países de América Latina en realidad se trata de elegir entre el camino de una aceptación pasiva sin reservas de todo aquello implicado en la globalización, o un camino 
diferente, que implique el despliegue de una capacidad nacional y/o regional para procesar las tendencias globales y ponerlas al servicio de las necesidades del desarrollo (Morales, Mercedes. El Proceso de Globalización, Coyuntura Histórica Inexorable). Para esto se hace necesario que el gobierno apoye de alguna manera a través del aporte de recursos económicos y direccione políticas y legislación que motiven el impulso y uso de las tecnologías de la información a nivel empresarial y de país, el cual conlleven una inclusión social, y en efecto, una cultura de la apropiación de estas, que deben ser acompañadas de procesos de investigación, gestión de conocimiento, estudios científicos, como elementos que fortalecen la transformación de las tecnologías. La era de inteligencia en redes está dando origen a una nueva economía, nuevas formas de hacer política y nuevas sociedades basadas en la digitalización. Esta transformación arrastra consigo a empresas y gobiernos, así como a individuos. (Tapscott, Don. La Economía Digital. 2002). Conforme a esta problemática, ¿qué ha hecho Colombia frente a esta situación?

La tecnología se ha hecho razón política. (Herber Marcuse. Ensayo "un hombre unidimensional).

\section{POLÍTICAS ECONOMICAS Y SOCIALES DE TI EN COLOMBIA FRENTE A LA GLOBALIZACIÓN}

Colombia ha desarrollado a nivel de gobierno políticas económicas y sociales con el fin de fortalecer las tecnologías en el país, como el CONPES 3834 el cual manifiesta lineamientos de política para estimular la inversión privada en ciencia, tecnología e innovación a través de deducciones tributarias (Consejo Nacional de Política Económica y Social, 2015). Igualmente, se encuentra el CONPES 3582 el cual hace referencia a la política nacional de ciencia, tecnología e innovación (Consejo Nacional de Política Económica y Social, 2009). Y otros que los complementan como el CONPES 3854 por el cual se da la política nacional de seguridad digital (Consejo Nacional de Política Económica y Social, 2016). Así mismo se encuentra la Estrategia de Gobierno Digital del Gobierno, y leyes asociadas como la Ley 1712 de 2014 en el que se regula la Transparencia y del Derecho de Acceso a la Información Pública Nacional (Congreso de la República de Colombia, 2014), la Ley 1581 de 2012 por el cual se reglamenta la protección de datos personales (Congreso de la República de Colombia, 2012), Ley 1273 de 2009 de la protección de la información y de los datos"- y se preservan integralmente los sistemas que utilicen las tecnologías de la información y las comunicaciones, entre otras disposiciones (Congreso de la República de Colombia, 2009), Ley 527 de 1999 se define y reglamenta el acceso y uso de los mensajes de datos, del comercio electrónico y de las firmas digitales (Congreso de la República de Colombia, 1999), entre otras que fortalecen y controlan el uso de las tecnologías de la información en Colombia.

Sin embargo, de acuerdo a lo manifestado por el Ministerio de Comercio, Industria y Turismo de Colombia la inversión estimada total, pública y privada, en ciencia y tecnología es del 0,38 por ciento del PIB actualmente, donde solo lo que se invierte en investigación y desarrollo científico y tecnológico equivale al 0,2 por ciento del PIB, lo que sea insuficiente para competir en el TLC. Entre las desventajas que más resalta la directora científica de la Corporación para Investigaciones Biológicas, Ángela Restrepo, en materia de ciencia y tecnología, están la falta de compromiso estatal y la falta de apoyo a los investigadores para que sus propuestas sean verdaderamente competitivas. Además, se debe repatriar la diáspora científica y vincularla a los grupos colombianos, pero con ofertas salariales ventajosas. Igualmente, se manifiesta que Los cerca de 180.000 millones de pesos que dedica cada año el Estado colombiano para investigación científica y tecnológica se quedan cortos para generar mayor competitividad y poder enfrentar los tratados comerciales (Ministerio de Comercio de Industria y Turismo. Publicación Inversiones en ciencia y tecnología son insuficientes para competir en el TLC: Colciencias). Así mismo, Don Tapscott habla que La Economía Digital trae consigo numerosas promesas y oportunidades ilimitadas para la creación de riqueza y desarrollo social. A la vez, implica peligro potencial - para aquellos individuos, empresas o sociedades que se queden atrás, el castigo puede ser inclemente. (Tapscott, Don. La Economía Digital. 2002). Lo anterior, en nuestra opinión consideramos que no solo basta disponer de planes de gobierno, políticas, leyes, entre otros elementos, sino se tiene un apoyo económico significativo que impulse a la sociedad a emerger en las tecnologías de la información con el propósito de ser competitivos, sostenibles y continuar evolucionando al nivel de los países desarrollados y de la globalización.

Así mismo, se hace necesario la formación y capacitación que contribuyan a desarrollar una 
cultura en el uso de las tecnologías de la información en Colombia. La institución debe formar competencias que sean el desarrollo de políticas y programas de e-administración, que impulsen la transformación, el fortalecimiento institucional y la eficiencia del Estado en su faceta de servicio al ciudadano y a las empresas, empleando al máximo el potencial que ofrecen las TIC. (Banco de Desarrollo de América Latina. Hacia la Transformación Digital de América Latina: Las Infraestructuras y los Servicios TIC en la Región. 2013).

Actualmente, las empresas a nivel mundial han estado a la vanguardia de las tecnologías y las comunicaciones para surgir en el mercado y no desaparecer de la competitividad. Aunque Colombia no se identifica como un país altamente desarrollado las organizaciones han empezado a invertir para no desvanecerse y poder ser competitivas. Sin embargo, para que estas inversiones sean fructíferas se debe capacitar al recurso humano y así aprovechar al máximo las oportunidades que el mercado les está brindando.

Colombia en los últimos años en razón de ser competitiva y sostenible en el mercado internacional se ha sometido a un cambio significativo a nivel organizacional y de país en el cual a través del desarrollo de políticas económicas enfocadas en las tecnologías de la información ha logrado implementar proyectos tecnológicos enfocados en infraestructura y capacitación que contribuyan a la disminución de la brecha digital. Así mismo, ha logrado impactar a la sociedad especialmente en regiones donde no llegaba la tecnología con herramienta e infraestructura que les permita disponer de una conectividad que la incorpore a poder comunicarse con más facilidad entorno a sus necesidades y que la conlleve al mundo de la globalización. Dicho impacto, desde ese punto de vista ha sido positivos, sin embargo, la capacitación y formación del uso de las tecnologías sigue siendo un factor determinante que contribuya a una mejor transformación digital en este camino de disrupción que se vive con las tecnologías. De igual manera, algunos creen que las tecnologías pueden traer perjuicios al verse amenazados por la misma al pensar que pueden perder sus empleos y ser reemplazados por una máquina. Igualmente, a nivel empresarial se puede pensar que la globalización de las tecnologías puede conllevar a la empresa a tener más competencia en el mercado. Por tal motivo, es muy importante que el gobierno en sus políticas mantenga programas de capacitación y formación efectivos que le permitan al ciudadano y a la empresa cambiar totalmente su pensamiento en torno del uso de las tecnologías en sus actividades $\mathrm{y}$ ver un cambio positivo que lo conlleva a su desarrollo interno y mundial. De esta manera, se comienzan a ver beneficios en la sociedad colombiana de conectividad, acercamiento entre regiones $y$ personas a nivel nacional e internacional, expansión de negocios, conocimientos de culturas, crecimiento empresarial, mejoramiento de la calidad en sus procesos, entre muchos más beneficios, que terminan por satisfacer las necesidades de la industria y de la sociedad.

\section{CONCLUSIONES}

Disponer de una estructura política, económica, social y cultural enfocada en la globalización que motive a la empresa colombiana y en general al país es fundamental para lograr posicionarse y mantenerse en el mercado a nivel nacional e internacional. Sin embargo, es importante el apoyo económico sea significativo para la implementación y uso de las tecnologías en Colombia.

Las empresas con una visión global en el mercado se vuelven más competitivas dependiendo el nivel tecnológico que adquieran e implementen en sus negocios.

La cultura en el uso de las tecnologías de la información es un eje primordial para incursionar en la transformación y disrupción tecnológica entorno a ser más competitivos y sostenibles conforme a las exigencias de la globalización de los mercados.

\section{REFERENCIAS}

Barroso, Paola (2009). Globalización.

Henao, Lara. Laura Alejandra (2013). Globalización: Amenaza y Oportunidad para las Naciones en desarrollo.

Beldarrain, Vicente (2012). Tecnología y Globalización Económica.

SH. Sunarno, Jakarta (2001). Globalization and Information Technology: Forging New Partnerships in Public Administration.

Christensen, Clayton (1997). The innovator's dilemma". Harvard Business Review Press.

Christensen, Clayton. Baumann, Heiner. Ruggles, Rudy y Sadtler Thomas M. (2006). Innovación 
disruptiva para el cambio social. Harvard Business Review Press.

Bruno Estrada López (2016). Disrupciones tecnológicas y empleo: Diagnostico y propuesta.

Morales, Mercedes. El Proceso de Globalización, Coyuntura Histórica Inexorable).

Tapscott, Don. La Economía Digital (2002). Marcuse, Herber. Ensayo "un hombre unidimensional.

Ministerio de Comercio de Industria y Turismo (2017). Publicación Inversiones en ciencia y tecnología son insuficientes para competir en el TLC: Colciencias.

Banco de Desarrollo de América Latina (2013). Hacia la Transformación Digital de América Latina: Las Infraestructuras y los Servicios TIC en la Región.

Clayton J Torres, John F Archila, Mario L Tronco, Marcelo Becker, Arthur J Viera Porto, Alexander J Tiberti (2013). Estudio cinemático de una plataforma robótica para agricultura. Revista colombiana de Tecnologías de Avanzada ISSN: 1692-7257 - Volumen 2 - Número 22.

Algemiro A. Ávila G, Elkin G. Flórez Serrano, Oscar Gualdrón G (2012). Diseño e implementación de un curso interactivo multimedia para el aprendizaje de los procesos CAM en un centro de mecanizado Leadwell V30. Revista Colombiana de Tecnologías de Avanzada ISSN: 1692-7257 - Volumen 2 - Número 20.

Luis A Mesa Mesa, Nelson Barrera Lombana (2013). La robótica educativa como instrumento didáctico alternativo en educación básica. Revista Colombiana de Tecnologías de Avanzada ISSN: 1692-7257 - Volumen 2 - Número 22.

Jaime Guzmán-Luna, Ingrid-Durley Torres, Juan Felipe Alvarez (2014). Propuesta de un generador de aplicaciones educativas basadas en televisión digital usando arquitectura de cómputo en la nube. Revista Colombiana de Tecnologías de Avanzada ISSN: 1692-7257 - Volumen 2 - Número 24.

Luis Fernando Gélvez R (2012). Aplicación de redes neuronales morfológicas al reconocimiento de vocablos simples. Revista Colombiana de Tecnologías de Avanzada ISSN: 1692-7257 Volumen 1 - Número 19. 